\title{
e-Phaïstos
}

e-Phaïstos Revue d'histoire des techniques / Journal of the history of technology

IX-1 | 2021

Autour de Léonard de Vinci

\section{Le marégraphe de Marseille : mesure, patrimoine, climat}

The Marseille Tide Gauge: Measurement, Heritage, Climate

\section{Alain Coulomb}

\section{(2) OpenEdition}

Journals

Édition électronique

URL : https://journals.openedition.org/ephaistos/8716

DOI : 10.4000/ephaistos.8716

ISSN : 2552-0741

Éditeur

IHMC - Institut d'histoire moderne et contemporaine (UMR 8066)

\section{Référence électronique}

Alain Coulomb, «Le marégraphe de Marseille : mesure, patrimoine, climat », e-Phaïstos [En ligne], IX-1 | 2021, mis en ligne le 27 avril 2021, consulté le 16 septembre 2021. URL : http://

journals.openedition.org/ephaistos/8716 ; DOI : https://doi.org/10.4000/ephaistos.8716

Ce document a été généré automatiquement le 16 septembre 2021.

Tous droits réservés 


\title{
Le marégraphe de Marseille : mesure, patrimoine, climat
}

The Marseille Tide Gauge: Measurement, Heritage, Climate

\author{
Alain Coulomb
}

1 Cet article a pour but de participer au rayonnement du marégraphe de Marseille, observatoire du niveau de la mer unique au monde, qui allie patrimoine et haute technicité depuis la fin du XIX ${ }^{e}$ siècle. Il fait appel à diverses notions qu'il n'a pas la prétention de traiter en profondeur: la marégraphie, le nivellement de précision, l'altimétrie... L'approche qui en est faite sous le prisme du marégraphe de Marseille espère aussi susciter chez le lecteur l'envie de mieux connaître ces disciplines scientifiques et techniques.

2 Les plus anciennes mesures du niveau de la mer ont été obtenues au moyen de planches graduées fixées le long de quais : les échelles de marées. Puis l'évolution des techniques a donné naissance aux marégraphes mécaniques. Les premiers sont apparus en Angleterre dans les années 1830 (Pouvreau 2008). En France, c'est l'ingénieur hydrographe Antoine Marie Rémi Chazallon (1802-1872) qui a inventé le mot marégraphe et réalisé les premières observations à Toulon, en 1844 (Pouvreau 2008).

3 Le mot marégraphe est composé de l'élément latin mare (la mer) et de l'élément grec grapho («j'écris », «je décris »). Étymologiquement, un marégraphe est un instrument qui trace l'évolution du niveau de la mer en fonction du temps. On appelle marégramme la courbe obtenue. Sur les appareils modernes, ce traçage ne se fait plus par un dessin mais au moyen de fichiers numériques. Le mot marégraphe désigne également le bâtiment qui abrite l'instrument de mesure. 
Fig.1. Bâtiments du marégraphe de Marseille

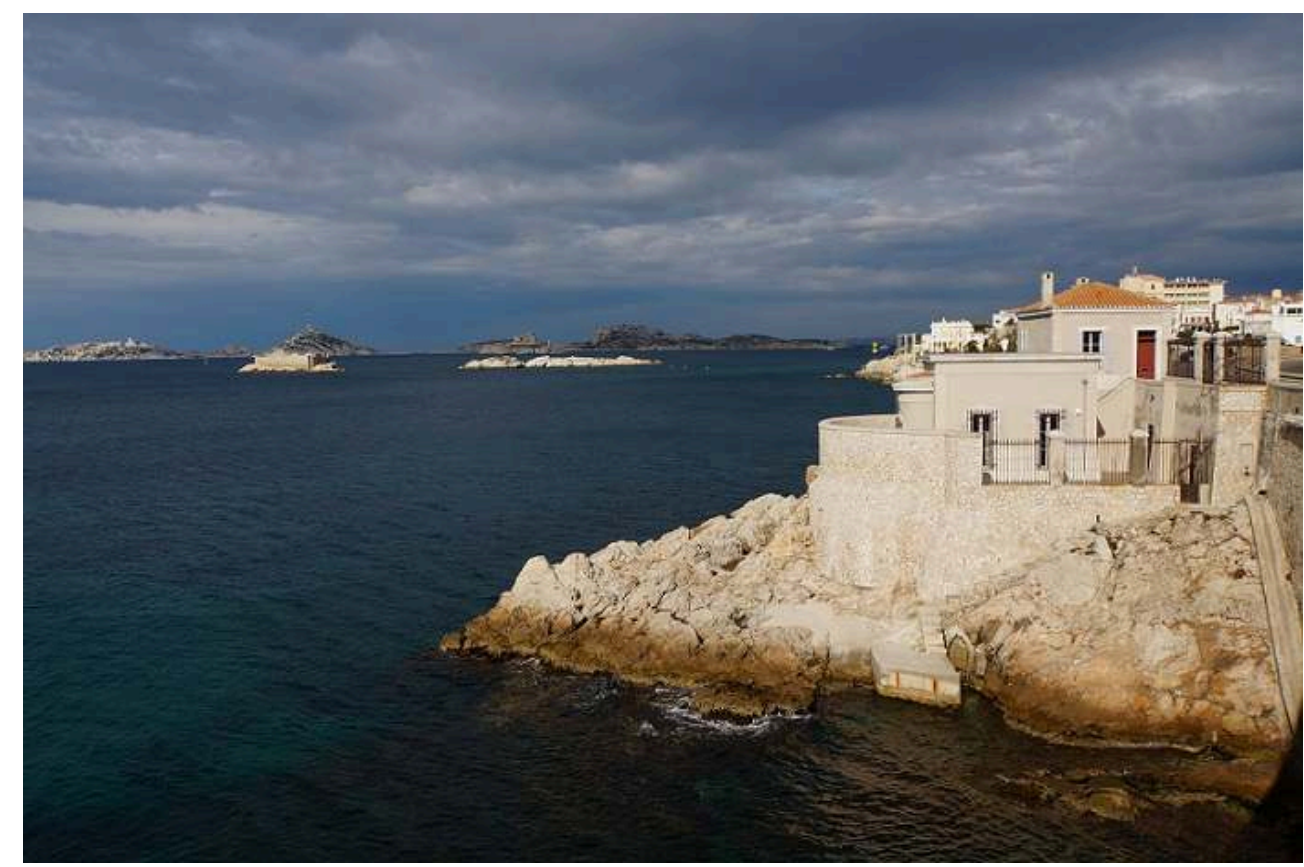

Les bâtiments du marégraphe de Marseille sont construits en 1883-1884 à moins de trois kilomètres du Vieux-Port, le long de la toute nouvelle promenade de la Corniche, une des plus élégantes de la cité phocéenne. L'ensemble immobilier comprend deux bâtiments principaux : un solide refuge pour l'appareil marégraphique et une maison d'habitation destinée à héberger le gardien des lieux (11 gardiens s'y sont succédé entre 1883 et 1988). Au premier plan, l'entrée de la galerie souterraine, taillée en forme d'ogive. Le marégraphe de Marseille a été édifié pour durer : il suffit d'apprécier le volume total de l'édifice et la qualité de sa construction pour s'en persuader. Sa construction ne relève pas de l'art d'un architecte mais du savoir-faire de Louis Auguste Sébillotte (1822-1888), conducteur des Ponts et Chaussées expérimenté qui remplissait les fonctions d'ingénieur.

(c) Alain Coulomb

4 Comment fonctionnaient les premiers marégraphes? Un marégraphe mécanique classique, c'est d'abord un flotteur, posé à la surface de la mer et dont la position évolue en fonction des mouvements verticaux de la mer. Par l'intermédiaire d'un câble métallique et de rouages, les mouvements de ce flotteur sont transmis à des instruments (crayons, plumes, etc.) qui tracent la courbe de la hauteur de la mer en fonction du temps. Le papier d'enregistrement s'enroule en effet sur un cylindre rotatif dont le moteur est une horloge. C'est la combinaison des mouvements de va-et-vient des instruments traceurs (en général une réduction des mouvements de la mer) et de la rotation du cylindre qui donne la sinusoïde de la courbe du niveau de la mer.

Le niveau de la mer dépend de la marée astronomique (l'attraction de la Lune essentiellement), phénomène donnant un résultat bien connu et prédictible, et de surcotes ou décotes provoquées par d'autres phénomènes (phénomènes météorologiques, climatiques, mouvements verticaux de l'écorce terrestre, etc.). Le terme surcote / décote désigne la différence entre le niveau marin observé et le niveau marin qui existerait en présence de la marée astronomique seule. En d'autres termes, la surcote/décote instantanée est la différence, à un instant $t$, entre la hauteur d'eau observée et la hauteur d'eau prédite. Il s'agit d'une surcote quand la hauteur d'eau observée est supérieure à la haute d'eau prédite ; on parle de décote dans le cas inverse (site internet Refmar). 
6 À Marseille, port situé au bord d'une mer à faible marée, l'effet produit sur le niveau de la mer par les variations météorologiques (la pression atmosphérique essentiellement, mais aussi les vents, la pluie, etc.) dépasse assez souvent l'effet produit par les mouvements des astres.

\section{Le nivellement de précision}

7 Aujourd'hui, les auteurs du Petit Larousse définissent très simplement, voire de manière simpliste, l'altitude d'un point de la surface terrestre comme «l'élévation verticale de ce point au-dessus du niveau de la mer». À l'Institut national de l'information géographique et forestière (IGN), l'altitude d'un point est définie comme «la coordonnée par laquelle on exprime l'écart vertical de ce point à une surface de référence proche du géoïde ».

8 Un géoïde est une surface de niveau (surface équipotentielle du champ de pesanteur) coïncidant au mieux avec le niveau moyen des mers. En raison des variations de la masse volumique de l'eau, des vents et des courants dominants, des variations de pression atmosphérique, la surface moyenne des mers n'est pas une surface équipotentielle. D'autre part, la façon dont le géoïde coïncide au mieux avec la surface moyenne des océans est ambiguë. Il s'ensuit que le géoïde ne peut pas être défini de manière univoque. Pour définir un géoïde, on peut convenir qu'il contient un point particulier, par exemple le niveau moyen enregistré par un marégraphe pendant une période donnée.

9 Malgré tous ces mouvements, et parce qu'elle supporte leurs embarcations, la mer présente une surface que les marins ont logiquement utilisé comme référence depuis très longtemps. Sur terre, il en a été tout autrement ! La mer est considérée dès le XIX ${ }^{\mathrm{e}}$ siècle, comme une surface de niveau; mais pour des raisons pratiques, elle est encore très peu utilisée comme surface de référence pour les opérations de nivellement. Quand les travaux sont éloignés du littoral, il est d'usage de fixer un plan de comparaison local. Bien souvent, le plan de comparaison est choisi au-dessus des points à coter. Ainsi, par exemple, «Sa Majesté ayant résolu de faire conduire à Versailles la meilleure eau pour boire que l'on pouvait trouver dans les lieux circonvoisins ", l'abbé Jean Félix Picard (1620-1682) est chargé par Louis XIV d'étudier les possibilités de réalisation d'un aqueduc permettant d'amener l'eau de la Loire au château. En habile courtisan, Picard choisit d'exprimer les cotes issues de ses nivellements par rapport au rez-de-chaussée de la demeure royale.

10 Puis des besoins d'unification commencent à se faire sentir. L'ingénieur Jean Antoine Fabre (1749-1834), auteur d'un Traité complet sur la théorie et la pratique du nivellement, écrit par exemple : «il serait nécessaire que le gouvernement fixât une seule et même ligne de niveau, ou plutôt une même et seule superficie horizontale dont la position fut connue et à laquelle on rapportât toutes les opérations de nivellement (...). Avec la moindre attention, on voit que la nature nous l'offre dans la superficie des eaux de la mer ». Fabre poursuit même son raisonnement en indiquant que la Méditerranée présente une surface plus stable que l'Océan et que, par conséquent, c'est la superficie de cette dernière mer qu'il faut prendre pour terme de comparaison.

11 Avec l'extension des travaux d'aménagement du territoire d'ampleur nationale, par exemple l'apparition du réseau des lignes de chemin de fer et, en parallèle à 
l'établissement de la carte d'État-major commencée au début du XVIII siècle, le besoin d'un nivellement général de la France se fait de plus en plus sentir. Les nombreux progrès techniques amenés par Paul Adrien Bourdalouë (1798-1868) dans l'art du nivellement rendent économiquement et techniquement possible son établissement. Cela se traduit dans le vocabulaire. Au XVIII ${ }^{e}$ siècle, la donnée altimétrique chiffrée est désignée par les termes hauteur, élévation ou plus souvent cote. Le nom altitude est absent de la sixième édition du Dictionnaire de l'Académie, éditée en 1835. L'altitude apparaît dans la littérature technique à partir du moment où l'on envisage le premier nivellement général de la France.

De nos jours, l'appellation Nivellement général de la France est générique et concerne trois réseaux de nivellement de grande ampleur qui se sont succédé sur le sol de la France continentale :

- Entre 1857 et 1864, Paul Adrien Bourdalouë établit un premier réseau national de nivellement. L'origine de ce réseau est le trait $0,40 \mathrm{~m}$ de l'échelle de marée du Fort Saint-Jean à Marseille. Les documents anciens semblent montrer que les calculs de Bourdalouë ne font pas intervenir de mesures de pesanteur (nous verrons que ce paramètre sera par la suite pris en compte pour obtenir des altitudes plus rigoureuses). - Dès 1878, le ministère des Travaux publics décide de poursuivre l'œuvre de Bourdalouë. Le travail est confié à un service public dirigé par Charles Lallemand (Closier 1937). L'origine de ce nouveau réseau est déterminée à l'issue d'observations du niveau de la mer réalisées au marégraphe de Marseille entre 1885 et 1897. Les altitudes dites orthométriques de ce réseau prennent en compte des valeurs de pesanteur théorique.

- Entre 1962 et 1969, l'IGN (à l'époque Institut géographique national) remet en état le réseau de base, en conservant l'origine du réseau NGF/Lallemand. Aujourd'hui encore, le système de référence vertical officiel est le NGF/IGN69, basé sur ces observations de nivellement faites dans les années 60 (il y a aussi un réseau NGF/IGN78 établi en Corse en 1978, dont l'origine est le niveau moyen de la mer à Ajaccio). Les nouvelles altitudes étant de type normal, elles prennent en compte un modèle utilisant des mesures de pesanteur réelle. Les différences entre le réseau NGF/Lallemand et NGF/IGN1969 progressent vers le Nord (les altitudes NGF/IGN69 sont supérieures aux altitudes NGF/ Lallemand, de $60 \mathrm{~cm}$ à Dunkerque et d'environ $33 \mathrm{~cm}$ à Paris).

Le nivellement est notamment utile pour les travaux d'aménagement du territoire. Pour rattacher précisément ses chantiers à la référence verticale nationale, le géomètre utilise des repères de nivellement implantés par l'IGN. Un repère de nivellement est un élément métallique dont l'altitude est déterminée avec une précision millimétrique. Plus de 400000 repères sont répartis sur le territoire national. Leurs données descriptives sont accessibles par le moyen d'une fiche signalétique consultable et téléchargeable sur internet (voir lien en webographie).

Pour compléter ces deux sites et satisfaire l'utilisateur mobile opérant sur le terrain, l'IGN a développé l'application Géodésie de poche, également téléchargeable (voir lien en webographie). 


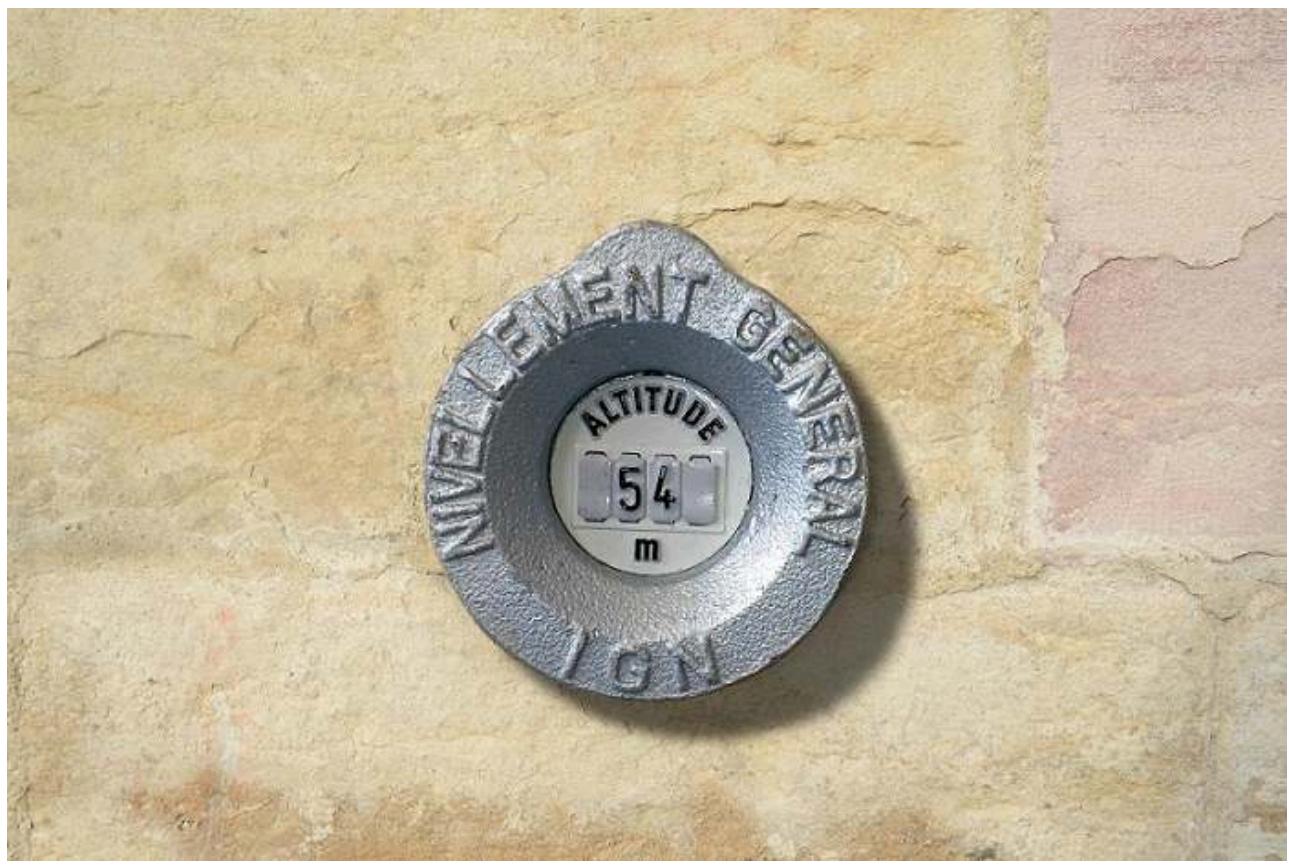

Il existe de nombreux types de repères de nivellement (médaillon, Bourdalouë, console, etc.). Certains repères comportent des plaquettes où peuvent figurer un matricule ou une altitude. Ces renseignements, s'ils présentent parfois un intérêt esthétique et patrimonial, ne sont plus d'actualité dans la plupart des cas. Pour connaître le matricule et l'altitude d'un repère de nivellement, il est indispensable de consulter sa fiche signalétique. Une plaquette en aluminium est fixée au centre des repères de type médaillon dont l'altitude a été déterminée ou contrôlée récemment (photo). Dans un but d'information d'un public non technicien, une altitude arrondie au mètre est fournie sur cette plaquette. Un repère qui n'est pas muni de plaquette n'est pas un repère abandonné. II est aussi utile qu'un repère disposant d'une plaquette et doit faire l'objet des mêmes mesures de conservation.

(c) IGN

\section{Le marégraphe totalisateur de Marseille}

15 L'instrument historique qui équipe l'observatoire marégraphique de Marseille est le résultat d'une collaboration intellectuelle de plus de six mois entre l'ingénieur allemand Heinrich Reitz, concepteur de ce type d'appareil, et l'ingénieur français Charles Lallemand (1857-1938), directeur du service du Nivellement général de la France, commanditaire de l'instrument. Cette collaboration visait en permanence l'excellence sans négliger l'esthétique. Reitz écrivait par exemple à Lallemand: "Afin que le surveillant ait à tout moment conscience de l'importance de cet appareil et prenne minutieusement soin de toutes les pièces ", l'instrument doit "afficher une certaine élégance». Sur un autre courrier, il insistait en ces termes: il est « indispensable que l'appareil inspire le respect au contrôleur ; ceci contribue (...) à ce que l'appareil soit utilisé avec le plus grand soin ». 


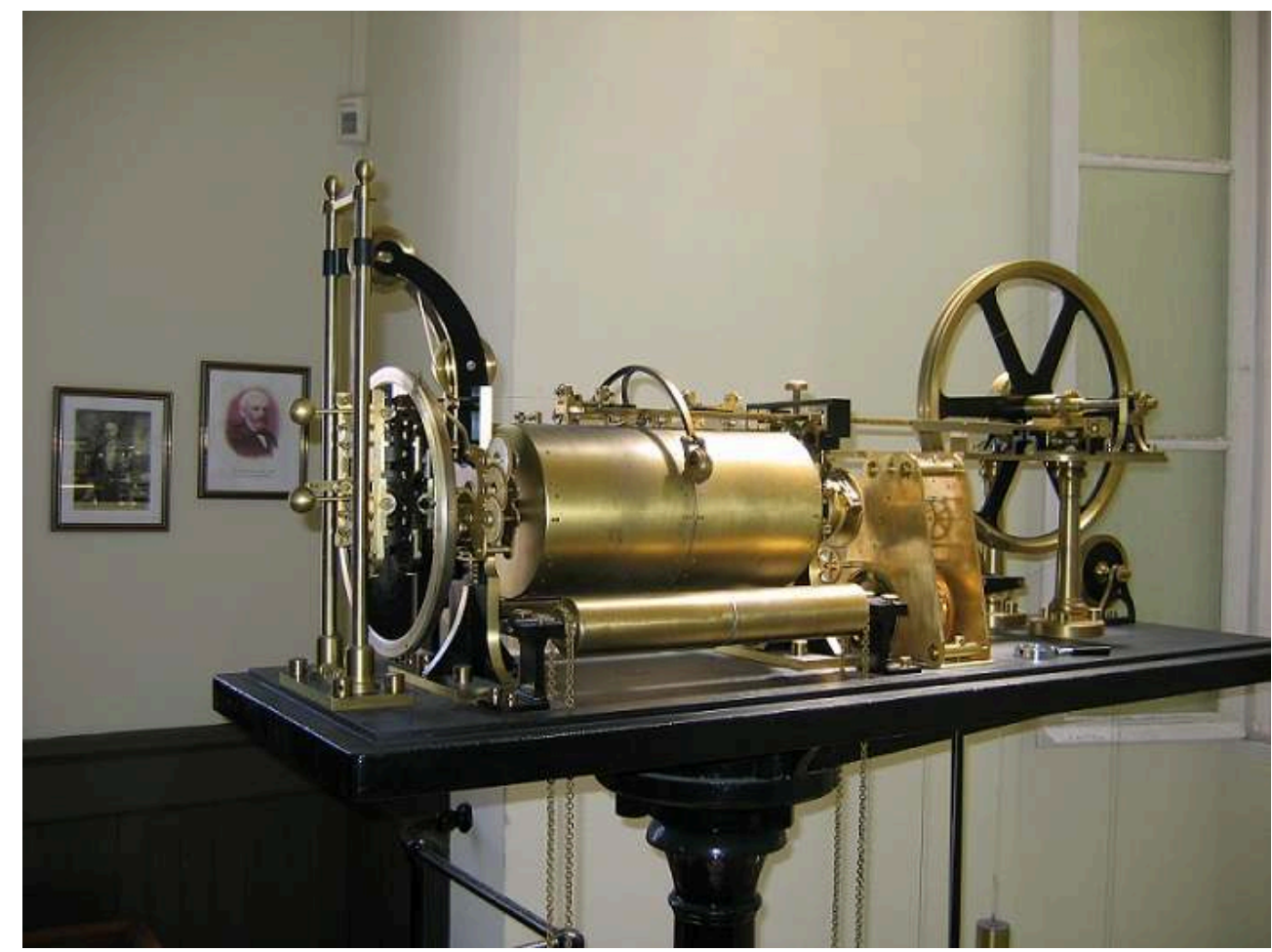

Depuis 1988 (départ du dernier gardien) l'appareil ne produit plus de marégramme. Seule la partie totalisatrice (à gauche de l'image) est encore utilisée. Cet appareil a été entièrement rénové en juin 2018. Les 10000 pièces qui le composent sont presque toutes en laiton. Le plateau du totalisateur (disque noir sur la partie gauche) est en verre. Les roulettes totalisatrices sont en agate.

(c) Alain Coulomb

La construction du marégraphe de Marseille donne lieu à plusieurs petites innovations technologiques telles que la compensation mécanique de l'allongement du câble de suspension du flotteur en fonction des variations de température, ou l'enregistrement en double des marégrammes, un exemplaire restant à Marseille pour les besoins du port, le second étant expédié au service du Nivellement général de la France.

17 Mais l'intérêt essentiel du marégraphe de Marseille réside surtout dans sa partie dite totalisatrice. Avant son invention, le niveau moyen sur une période de temps donnée pouvait être déterminé soit par un lourd calcul arithmétique, soit en mesurant sur les marégrammes, au moyen d'un planimètre, l'aire formée par la courbe du niveau de la mer, l'axe des temps et les deux droites figurant les bornes de la période considérée. De cette façon, le résultat était obtenu beaucoup plus rapidement mais manquait un peu de précision. Le marégraphe totalisateur remplace tout ce travail par une simple division de deux chiffres fournis par l'instrument et dont l'un est proportionnel au temps écoulé. Comme cette détermination directe se fait sans l'intermédiaire d'un diagramme, on obtient une précision extraordinaire, bien supérieure à celle obtenue avec un planimètre.

18 Comment fonctionne le marégraphe totalisateur? Le câble auquel est attaché le flotteur fait évoluer horizontalement une pièce appelée crémaillère (une barre horizontale entraînant les aiguilles qui traçaient, sur papier, le niveau instantané de la mer). Le papier d'enregistrement (le marégramme) est déroulé par un cylindre dont la rotation est régulière (il est mû par une horloge). 
Fig.4. L'horloge du marégraphe totalisateur

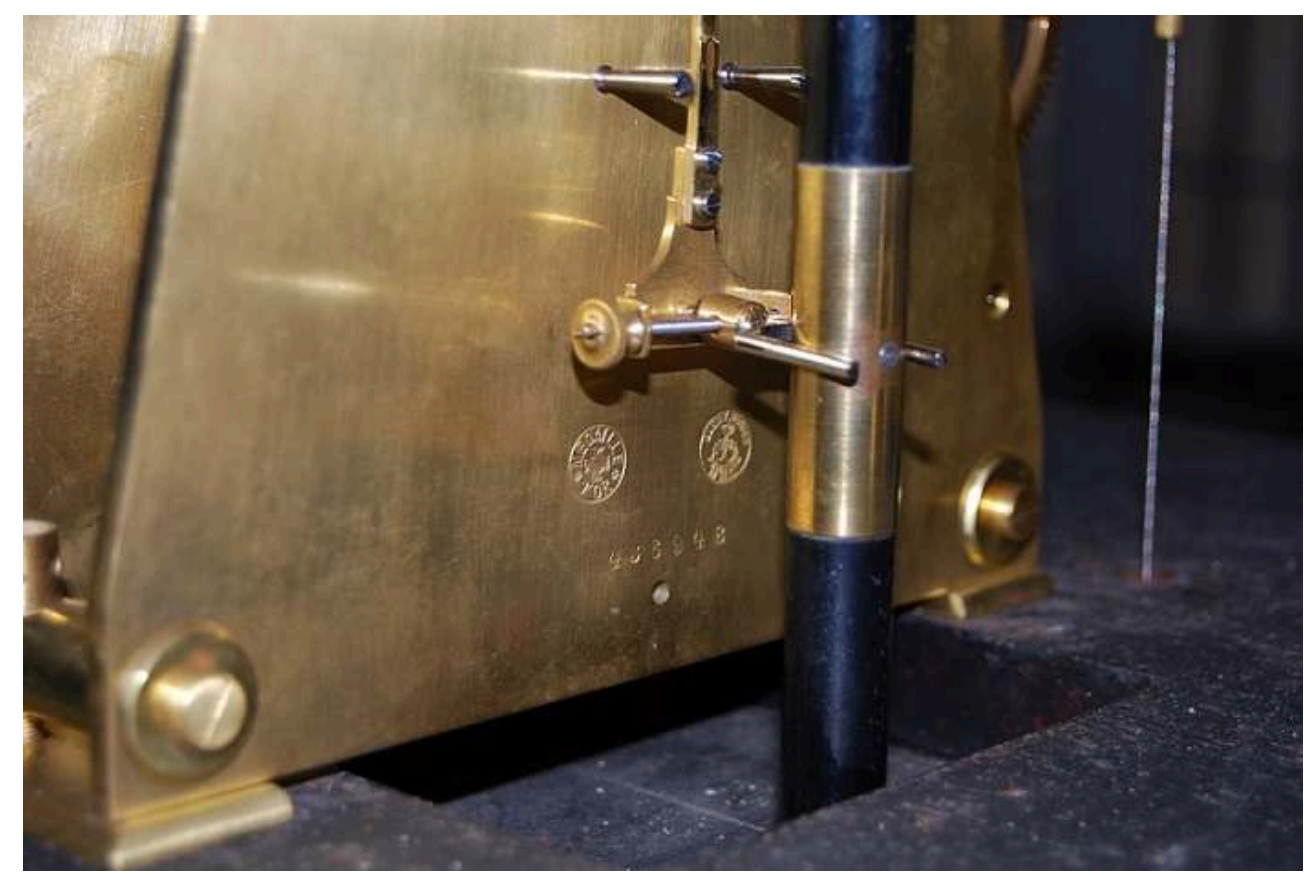

Le mécanisme d'horlogerie du Marégraphe de Marseille est dû au Prussien Gustav

Becker (1819-1885). Véritables ouvrages d'art, les mécanismes d'horlogerie Becker, connus pour leur qualité de fabrication, sont faciles à identifier. Ils portent la marque de l'entreprise, qui comprend la première médaille d'or acquise en 1852, les initiales et le nom de l'horloger, ainsi que le nom de la ville de Silésie qui a abrité sa première fabrique. Les numéros de série atteignent 260000 en 1880 et 500000 en 1885, celui du mécanisme du Marégraphe de Marseille, fabriqué en 1884, porte le $n^{\circ} 456948$

(c) Alain Coulomb

19 Le totalisateur est un intégrateur mécanique. Son mécanisme se compose d'un chariot porte-roulettes porté par deux perches verticales et d'un disque vertical en verre disposé sur le même axe que le cylindre d'enregistrement (et qui tourne à la même vitesse). Le chariot est relié à l'extrémité de la crémaillère par un mince ruban de platine, qui, en s'infléchissant sur deux poulies, transforme le mouvement de va-etvient horizontal de la crémaillère en un mouvement vertical alternatif de translation (quand le niveau de la mer descend, le chariot monte et inversement).

Deux roulettes en agate sont en contact avec le disque de verre et leur mouvement est créé par la rotation régulière du disque. Par un système d'engrenages, le mouvement de ces roulettes commande celui de cadrans gradués. L'incrémentation de leurs graduations dépend de la hauteur d'eau; elle est d'autant plus rapide que le cadran va vers l'extérieur du disque (en vertu de la proportionnalité de la vitesse avec la distance à l'axe de rotation), et d'autant plus lent qu'il va vers l'axe de rotation. C'est une manière de traduire mécaniquement les vitesses variables des changements du niveau de la mer (vitesse lente à l'étale, vitesse plus ou moins rapide en marée montante ou descendante).

21 Ainsi, si entre deux instants $t_{0}$ et $t_{1}\left(t_{1}>t_{0}\right)$, on mesure deux graduations $g_{0}$ et $g_{1}$ sur les cadrans $d u$ totalisateur, la graduation moyenne est simplement $\left(g_{1}-g_{0}\right) /\left(t_{1}-t_{0}\right)$. Mathématiquement, le totalisateur détermine l'intégrale de la fonction analogique 
qu'est le niveau de la mer, dont on déduit immédiatement la moyenne (Coulomb 2014a).

Fig.5. Principe de fonctionnement du marégraphe totalisateur

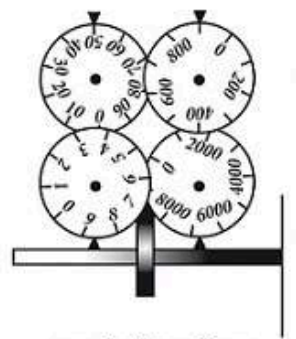

vue de face d'une des deux roulettes r1 ou r2 2 t de ses compteurs de tours

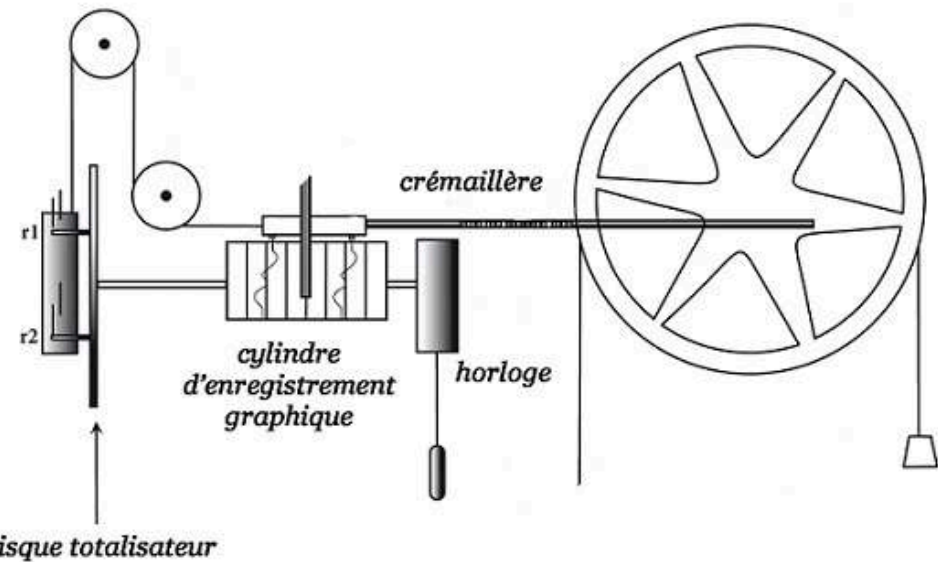

disque totalisateur

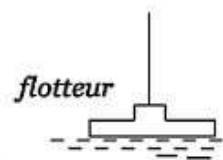

Le niveau moyen de la mer est obtenu par une simple division de deux chiffres fournis par l'instrument : une somme de niveaux d'eau et une durée. Le marégraphe est dit totalisateur parce qu'il fait en permanence des sommes de niveaux d'eau, lisibles sur les cadrans figurés à gauche du croquis. Pour éviter les erreurs et détecter les mauvais fonctionnements, il y a deux roulettes en contact avec le disque entraîné par l'horloge ; elles mesurent la même quantité. Chaque roulette r1 et r2 est équipée de 4 cadrans (un pour les unités, un pour les dizaines, un pour les centaines et un pour les milliers). Le temps écoulé est aussi lisible sur l'instrument, notamment au bord du disque de verre.

(c) Alain Coulomb

Reitz fait construire ses instruments par Johann Christian Dennert (1829-1920) et son collègue Martin Pape (1834-1884). Dennert et Pape assemblent trois marégraphes totalisateurs. Le premier est installé sur l'île d'Heligoland dans la mer du Nord, le deuxième à Cadix en Espagne, et le troisième à Marseille. Les deux premiers, non conçus pour durer, disparaissent assez rapidement. Celui de Marseille, amené sur place dans cinq caisses transportées par le steamer Hamburg et installé sur la Corniche par Christian Dennert en janvier 1885, est aujourd'hui en parfait état de marche (même si la partie enregistrement papier n'est plus utilisée depuis le départ du dernier gardien en 1988). Il est donc unique au monde.

L'arrêté du 28 octobre 2002 considère d'ailleurs que « la conservation du Marégraphe de Marseille (Bouches-du-Rhône) présente au point de vue de l'histoire un intérêt public en raison de l'importance de cette réalisation au regard de l'évolution des procédés de mesure scientifiques». Il classe l'observatoire (l'ensemble immobilier, l'appareil et les installations techniques) parmi les monuments historiques. 


\section{La fixation de l'origine du réseau NGF/Lallemand, devenue ensuite celle du réseau NGF/IGN69} février 1885 et le $1^{\text {er }}$ janvier 1897, mais il en est cependant extrêmement proche (Coulomb 2014a).

Fig.6. Fixation du zéro des altitudes françaises continentales

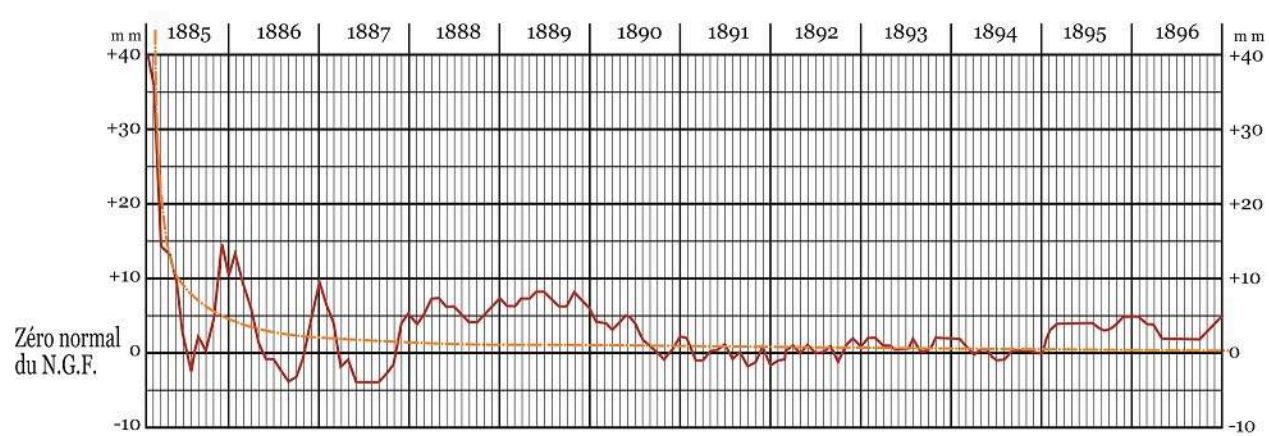

En rouge, la variation du niveau moyen de la mer, depuis le 3 février 1885 jusqu'au 1 er janvier 1897 ; en jaune, l'hyperbole équilatère dessinée par les ingénieurs du NGF.

(c) Alain Coulomb

Le zéro NGF/Lallemand, devenu ensuite le zéro NGF/IGN69, est établi exactement à 71 millimètres au-dessous du zéro de Bourdalouë.

\section{Qualités du zéro fixé au marégraphe de Marseille}

Le zéro fixé en 1897 est purement conventionnel : on aurait pu choisir un autre lieu ou une autre période et le résultat aurait pu être sensiblement différent.

Il est immuable, ou en tous cas il a été immuable jusqu'à nos jours : on sait que le niveau moyen actuel de la Méditerranée à Marseille n'est plus le même qu'à la fin du XIX ${ }^{\mathrm{e}}$ siècle; on ne change pas pour autant les altitudes des repères de nivellement. Cela ne servirait à rien : d'un point de vue opérationnel, ce qui est utile, ce n'est pas l'altitude de chaque point, c'est la différence de niveau qui existe entre les différents points.

Il est peu pratique car il n'est pas matérialisé (pas de trait gravé, pas de repère scellé au niveau 0 ) et il est difficile d'accès (dans un puits). C'est pour cette raison qu'existe la notion de repère fondamental. 


\section{Le repère fondamental du nivellement français continental}

30 sert de point de départ pratique aux mesures et aux calculs de toutes les altitudes d'un réseau de nivellement. L'altitude d'un point fondamental est en général fixée conventionnellement à partir des observations d'un marégraphe dont il est proche.

ère fondamental de Marseille est à 1,661m au-dessus du zéro de 1897. Il est scellé dans le bâtiment du marégraphe, dans des conditions particulièrement favorables de stabilité et de conservation. Il est constitué par un rivet en bronze dont la calotte supérieure est recouverte d'un alliage très dur de platine et d'iridium. Ce rivet est scellé dans un bloc cylindrique de granit, lui-même incrusté dans le rocher calcaire qui forme le promontoire sur lequel le marégraphe est construit.

Fig.7. Point fondamental du réseau de nivellement français continental

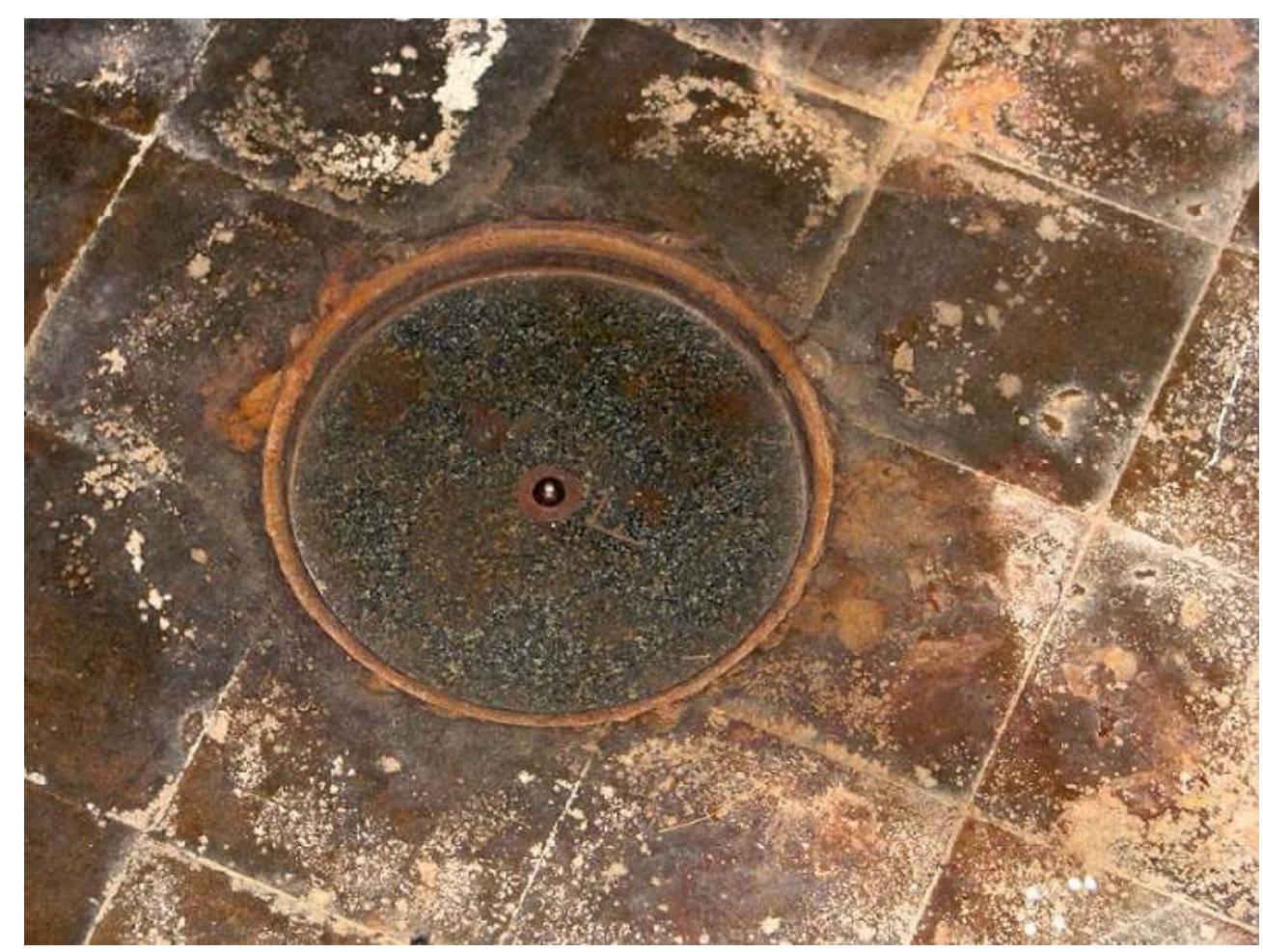

Ce point n'a pas été scellé volontairement à 1,661 m d'altitude. II a été implanté en 1884, au moment de la construction des bâtiments. Pendant douze ans, on a ensuite mesuré le niveau de la mer par rapport à ce repère fixe. Une fois le zéro fixé, son altitude s'est trouvée être 1,661 m. Plutôt que de dire que le point fondamental est à 1,661 m au-dessus du zéro, on peut dire que le zéro est à 1,661 m audessous du point fondamental. Le zéro de Marseille est aussi celui des altitudes suisses (la Suisse est un pays sans littoral), mais le point fondamental du nivellement suisse est implanté à Genève sur un rocher appelé Pierre du Niton.

(c) Alain Coulomb

\section{Liens entre zéro des altitudes et zéro hydrographique}

Le zéro des altitudes, établi à Marseille à la fin du XIX siècle, s'applique à toute la France continentale. Sur ce même territoire, le zéro hydrographique (référence des cartes marines et des annuaires de marée) n'est pas unique. Le souci des marins est avant tout la sécurité de la navigation. Le zéro hydrographique est voisin du niveau des 
plus basses mers astronomiques. Ce choix est commode, car le marin est ainsi assuré de disposer d'au moins autant d'eau que ce qui est indiqué sur la carte marine, quelle que soit l'amplitude de la marée (aux surcotes ou décotes près).

Pour chaque site français de mesure du niveau de la mer (observatoire de marée), le Shom (Service hydrographique national) tient à jour et diffuse le produit Références altimétriques maritimes (RAM) ou cotes des zéros hydrographiques par rapport aux systèmes de référence altimétriques légaux (NGF/IGN69 par exemple).

Pour chaque observatoire de marée, le Shom entretient aussi une fiche descriptive. Parmi les renseignements présents sur cette fiche figurent notamment la localisation les repères de nivellement proches de l'appareil de mesure, leur élévation par rapport au zéro hydrographique, si possible leur altitude... À Marseille, le zéro NGF/IGN69 est supérieur de $0,329 \mathrm{~m}$ au zéro hydrographique.

\section{Le marégraphe moderne}

\section{Le marégraphe côtier numérique}

Sur proposition du Conseil national français de géodésie et de géophysique (CNFGG), et de manière à mieux répondre aux spécifications internationales, l'IGN a, en juillet 1998, équipé l'observatoire de Marseille d'un marégraphe côtier numérique (MCN). Ce premier marégraphe numérique fonctionnant avec des ondes acoustiques a été remplacé par un instrument plus moderne en avril 2009.

Celui-ci fonctionne grâce à des ondes radar. L'instrument mesure le temps de parcours, selon la verticale, d'impulsions électromagnétiques réfléchies par la surface de la mer. Les fichiers des données acquises sont régulièrement transmis au Shom via le réseau téléphonique.

L'implantation d'un marégraphe est en effet souvent liée à des activités maritimes et la plupart des marégraphes français sont gérés par le Shom, opérateur en France du Réseau d'observation du niveau de la mer (RONIM) constitué de 50 marégraphes côtiers numériques.

Jusqu'en 1998, les moyennes mensuelles et annuelles collectées par le Permanent Service for Mean Sea Level (PSMSL), service scientifique international créé en 1933, étaient établies à partir des données fournies par le marégraphe totalisateur installé en 1885 . Depuis 1998, les données transmises au PSMSL sont celles du MCN.

Le marégraphe totalisateur n'en est pas pour autant mis à la retraite. Il est toujours entretenu et, comme le marégraphe numérique, fait l'objet d'un étalonnage annuel. Des mesures hebdomadaires y sont constamment effectuées, en parallèle des mesures faites par le marégraphe numérique. Le rôle du marégraphe mécanique est d'assurer la continuité des données de niveau de la mer (il ne faudrait pas que ce qui semble être une hausse du niveau soit en réalité due à un changement d'appareil ou de technique de mesure).

\section{La station GNSS permanente}

Les marégraphes de Marseille mesurent le niveau de la mer par rapport à des repères matériels implantés à l'intérieur des bâtiments. Quand le résultat de ces mesures donne 
une valeur qui diminue avec le temps, la question qu'il convient de se poser est : que mesure-t-on, une hausse du niveau de la mer, un tassement du point de référence, ou une combinaison de ces deux phénomènes?

Les informations transmises par les satellites GNSS (Global Navigation Satellite System), nom générique des systèmes de navigation satellitaires sur l'ensemble de la planète tels que GPS (États-Unis), GLONASS (Russie), Galileo (Union européenne), Beidou (Chine), peuvent répondre à ces questions. En recevant les signaux émis par les satellites GNSS, l'antenne GNSS installée sur le toit du marégraphe depuis 1998, couplée à un récepteur qui est à l'intérieur, détermine les mouvements absolus du bâtiment.

Dans le domaine altimétrique, l'exploitation des mesures GNSS rencontre deux difficultés :

- Les signaux GNSS traversent la troposphère, où les gouttelettes d'eau contenues dans les nuages ralentissent leur progression (on parle de délai troposphérique). Ce retard est difficile à estimer précisément.

- Les systèmes GNSS déterminent des hauteurs au-dessus d'une forme mathématique de la Terre : un ellipsoïde. Ils ne donnent pas directement une altitude (élévation audessus du géoïde). On passe de la hauteur ellipsoïdale (mesurée par GNSS) à l'altitude par l'intermédiaire d'une grille de conversion. Sur le territoire français continental, la grille de conversion altimétrique en vigueur s'appelle RAF18. Elle permet de déterminer des altitudes par observations GNSS avec une précision centimétrique $(1 \mathrm{~cm}$ à 95\%), à condition d'utiliser des méthodes GNSS précises et de se rattacher précisément à la référence géodésique RGF93, dont l'accès est facilité depuis la mise en place du réseau GNSS permanent (RGP) en 1998.

Néanmoins, malgré ces deux difficultés, des séries longues d'observations GNSS, établies au moyen de stations permanentes associées avec les marégraphes, offrent des résultats dont la précision est du même ordre que celle de la marégraphie. L'association des deux types de mesures a pour but de connaitre l'évolution réelle du niveau moyen de la mer. Les GNSS donnent une information sur le mouvement absolu du sol, les marégraphes sur les mouvements de la mer relatifs au sol, la combinaison des deux permettant de comprendre le comportement réel du niveau de la mer à l'endroit considéré.

\section{La numérisation des marégrammes de Marseille}

La sauvegarde des données fournies entre 1885 et 1988 par les marégrammes de Marseille (dont le papier se détériore avec le temps) et l'exploitation de ces données par des moyens informatiques a nécessité leur conversion sous un format compatible avec les ordinateurs. Ce travail colossal de numérisation de 1200 marégrammes a été effectué entre 1996 et 2001, grâce à une action concertée de l'IGN et du Shom.

Les marégrammes de Marseille sont en passe d'être classés monuments historiques, alors qu'aujourd'hui seuls l'ensemble immobilier, l'appareil et les installations techniques sont concernés par l'arrêté de 2002. Ce classement doit s'accompagner de mesures de protection supplémentaires. 


\section{La série temporelle du marégraphe de Marseille}

La série temporelle de mesures fournie par le marégraphe de Marseille est très longue (135 ans d'observations, pratiquement sans interruption). Cette série est également l'une des plus « cohérente » (très peu de changements d'appareils, pratiquement pas de changements dans l'environnement des mesures; etc.). Elle permet donc d'établir une tendance robuste et fiable, qui semble linéaire : à Marseille, l'élévation du niveau de la Méditerranée depuis 1885 est d'environ 16 centimètres; le rythme est d'environ $1,2 \mathrm{~mm} / \mathrm{an}$.

Fig.8. Élévation du niveau de la mer à Marseille établie à partir des moyennes annuelles calculées et validées par le PSMSL

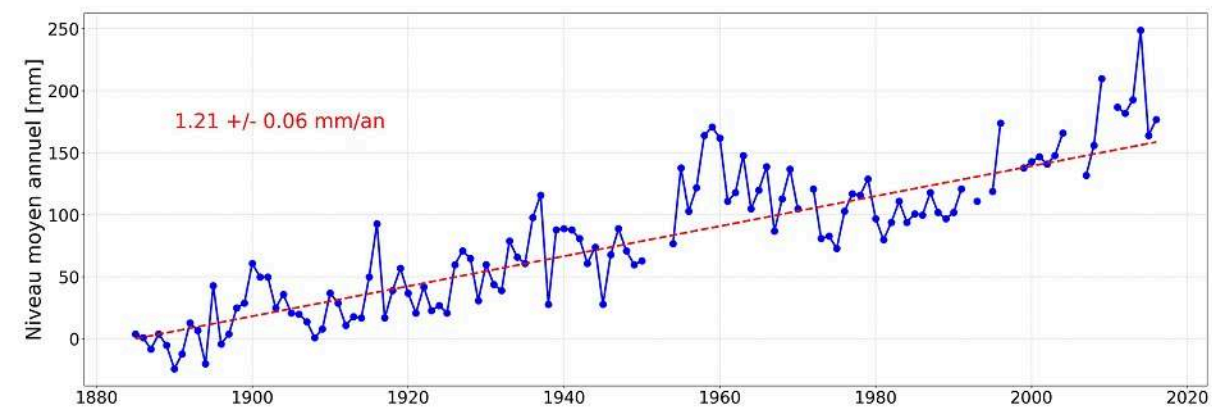

La courbe présente des lacunes car, quand il manque plus de 15 jours d'observations dans le mois, la moyenne mensuelle n'est pas calculée ; de même, il faut au moins 11 moyennes mensuelles par an pour que la moyenne annuelle soit calculée. Une courbe analogue, établie à partir de moyennes mensuelles, présenterait moins de lacunes et donnerait donc une tendance différente $(1,30 \pm 0,04 \mathrm{~mm} / \mathrm{an})$, statistiquement cohérente avec la tendance de la courbe ci-dessus. Une courbe établie à partir de moyennes mensuelles et prenant en compte 18 mois de mesures réalisées entre 1849 et 1851 au port de la Joliette, à environ 2,5 km du marégraphe totalisateur, donnerait une autre tendance encore légèrement différente $(1,26 \pm 0,04 \mathrm{~mm} / \mathrm{an})$, statistiquement cohérente avec les deux premières (Woppelmann et al., 2014)

(c) Médérix Gravelle - SONEL

La qualité de la série temporelle de Marseille a attiré l'attention de la Commission Océanographique Intergouvernementale (COI) de l'UNESCO qui a mis en œuvre un réseau mondial permanent d'observatoires du niveau de la mer connu sous le nom de Global Sea Level Observing System (GLOSS). Les marégraphes de Marseille et de Brest sont intégrés à GLOSS, réseau composé de plusieurs centaines de marégraphes formant une ossature autour de laquelle se rattachent des projets plus denses, régionaux ou nationaux comme RONIM.

\section{La valorisation scientifique}

L'ensemble immobilier du marégraphe de Marseille appartient à l'État qui, par convention, le met à disposition de l'IGN pour «mettre en œuvre l'infrastructure géodésique nationale, entretenir la référence géographique et altimétrique du territoire français, valoriser la culture et la technique des sciences géomatiques (notamment en ouvrant le site au public)». 

exemplaire, par le volume et la qualité architecturale de son ensemble immobilier, par la qualité exceptionnelle d'une partie de ses enregistrements graphiques, le marégraphe de Marseille est unique au monde. Bel exemple d'un continuum temporel de la science et des techniques, il n'est pas seulement un lieu chargé d'histoire ; il est aussi une station de surveillance de haute qualité, équipée d'appareils modernes qui en font un observatoire essentiel des programmes nationaux et internationaux d'observation du niveau des mers. Le principal intérêt actuel du marégraphe de Marseille réside dans sa participation à la compréhension et au suivi des changements climatiques. L'importance des conséquences de ces changements est et sera telle qu'elle justifie que les actions de valorisation engagées depuis quelques années soient amplifiées et rendues plus innovantes. Dans ce but, de nombreuses personnes d'horizons différents, constatant que le marégraphe de Marseille présente un intérêt spécifique et remarquable, ont d'ailleurs décidé de créer une association pour le faire mieux connaître. L'association Les amis du marégraphe de Marseille a pour objet de réunir des personnes physiques ou morales souhaitant agir pour la mise en valeur, la promotion, le renom et le rayonnement du marégraphe de Marseille. Elle utilise tous les moyens à sa disposition pour présenter le marégraphe de Marseille, son histoire et son intérêt scientifique actuel. L'association se propose d'établir et de conforter des liens avec d'autres acteurs du patrimoine et de la science ${ }^{1}$. 


\section{BIBLIOGRAPHIE}

CLOSIER René, « Le service du nivellement général de la France », L'information géographique, vol.

$2, \mathrm{n}^{\circ} 1,1937$, p. $14-15$

COULOMB Alain, 2014a, Le marégraphe de Marseille. De la détermination de l'origine des altitudes au suivi des changements climatiques : 130 ans d'observation du niveau de la mer, Presses des Ponts \& Chaussées, 2014, ISBN : 978-2-85978-481-2

COULOMB Alain, 2014b, « Contrôle des marégraphes de l'observatoire de Marseille », Document interne IGN IT/G276 - V3 du 15 juin 2014

POUVREAU Nicolas, Trois cents ans de mesures marégraphiques en France : outils, méthodes et tendances des composantes du niveau de la mer au port de Brest, Thèse de doctorat de l'Université de La Rochelle soutenue le 26 septembre 2008, HAL Id : tel-00353660

WOPPELMANN Guy, MARCOS Marta, COULOMB Alain, MARTIN-MIGUEZ Belen, BONNETAIN Paul, BOUCHER Claude, GRAVELLE Médéric, SIMON Bernard et TIPHANEAU Pascal, « Rescue of the historical sea level record of Marseille (France) from 1885 to 1988 and its extension back to 1849-1851 », Journal of Geodesy, September 2014, DOI: 10.1007/s00190-014-0728-6

\section{Webographie}

http://geodesie.ign.fr

https://geodesie.ign.fr/index.php?page=geodesie-poche

https://www.psmsl.org/

http://refmar@shom.fr

http://rgp.ign.fr

\section{NOTES}

1. Pour contacter l'Association des amis du marégraphe : amis.maregraphe@gmail.com.

\section{RÉSUMÉS}

Le marégraphe de Marseille a été construit à la fin du dix-neuvième siècle pour fixer le zéro des altitudes françaises continentales. Son existence est donc liée à la technique du nivellement de précision, ensemble d'opérations chiffrées qui permettent de traduire le relief. L'évoquer conduit plus généralement à parler de géodésie, science qui mesure la forme et les dimensions de la Terre. Le marégraphe de Marseille n'est pas seulement un lieu chargé d'histoire, c'est également une station de surveillance équipée d'appareils modernes qui en font un observatoire essentiel des programmes nationaux et internationaux d'observation du niveau des mers, niveau 
aujourd'hui à la fois dépendant et révélateur des changements climatiques. Le marégraphe de Marseille est enfin un lieu de vulgarisation scientifique dont l'activité mérite d'être conservée et développée.

The Marseille tide gauge was built at the end of the nineteenth century to fix the zero for continental French altitudes. Its existence is therefore linked to the technique of precision leveling, a set of quantitative operations that represent the relief. More generally, it falls within geodesy, a science that measures the shape and dimensions of the Earth. The Marseille tide gauge is not only a place steeped in history, it is also a monitoring station equipped with modern devices that make it an essential observatory of national and international programs for observing sea level which is both dependent on and indicative of climate change. The Marseille tide gauge is finally a place of popular science whose activity deserves to be preserved and developed.

\section{INDEX}

Mots-clés : histoire des techniques, histoire des sciences, nivellement, géodésie, océanographie, patrimoine scientifique, instrument scientifique, culture scientifique et technique, données

Thèmes : Un objet une technique

Keywords : history of technology, history of science, geodesing, oceanography, scientific heritage, data

\section{AUTEUR}

\section{ALAIN COULOMB}

Alain Coulomb est ingénieur à l'Institut national de l'information géographique et forestière (IGN). Il dirige le département qui entretient notamment le réseau de Nivellement général de la France (NGF) et contrôle le bon fonctionnement des instruments du marégraphe de Marseille. Il partage sa passion pour cet observatoire du niveau de la mer avec les lecteurs de son livre et les visiteurs du marégraphe. 